\title{
Weak Hard X-Ray Emission from Broad Absorption Line Quasars: Evidence for Intrinsic X-Ray Weakness
}

\author{
Luo, B.; Brandt, W. N.; Alexander, D. M.; Stern, D.; Teng, S. H.; Arevalo, P.; Bauer, F. E.; Boggs, S. E.; \\ Christensen, Finn Erland; Comastri, A.
}

Total number of authors:

22

Published in:

Astrophysical Journal

Link to article, DOI:

10.1088/0004-637X/794/1/70

Publication date:

2014

Document Version

Publisher's PDF, also known as Version of record

Link back to DTU Orbit

Citation (APA):

Luo, B., Brandt, W. N., Alexander, D. M., Stern, D., Teng, S. H., Arevalo, P., Bauer, F. E., Boggs, S. E., Christensen, F. E., Comastri, A., Craig, W. W., Farrah, D., Gandhi, P., Hailey, C. J., Harrison, F. A., Koss, M., Ogle, P., Puccetti, S., Saez, C., ... Zhang, W. W. (2014). Weak Hard X-Ray Emission from Broad Absorption Line Quasars: Evidence for Intrinsic X-Ray Weakness. Astrophysical Journal, 794(1), [70].

https://doi.org/10.1088/0004-637X/794/1/70

\section{General rights}

Copyright and moral rights for the publications made accessible in the public portal are retained by the authors and/or other copyright owners and it is a condition of accessing publications that users recognise and abide by the legal requirements associated with these rights.

- Users may download and print one copy of any publication from the public portal for the purpose of private study or research.

- You may not further distribute the material or use it for any profit-making activity or commercial gain

- You may freely distribute the URL identifying the publication in the public portal 


\title{
WEAK HARD X-RAY EMISSION FROM BROAD ABSORPTION LINE QUASARS: EVIDENCE FOR INTRINSIC X-RAY WEAKNESS
}

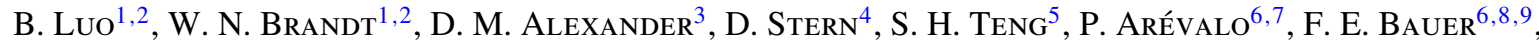 \\ S. E. Boggs ${ }^{10}$, F. E. Christensen ${ }^{11}$, A. Comastri ${ }^{12}$, W. W. Craig ${ }^{10,13}$, D. Farrah ${ }^{14}$, P. Gandhi ${ }^{3}$, \\ C. J. Hailey ${ }^{15}$, F. A. Harrison ${ }^{16}$, M. Koss ${ }^{17}$, P. Ogle ${ }^{18}$, S. Puccetti ${ }^{19,20}$, C. SAeZ ${ }^{21}$, A. E. ScotT ${ }^{1,2}$, \\ D. J. WaLton ${ }^{16}$, AND W. W. Zhang ${ }^{22}$ \\ ${ }^{1}$ Department of Astronomy \& Astrophysics, 525 Davey Lab, The Pennsylvania State University, University Park, PA 16802, USA \\ ${ }^{2}$ Institute for Gravitation and the Cosmos, The Pennsylvania State University, University Park, PA 16802, USA \\ ${ }^{3}$ Department of Physics, Durham University, South Road, Durham DH1 3LE, UK \\ ${ }^{4}$ Jet Propulsion Laboratory, California Institute of Technology, Pasadena, CA 91109, USA \\ ${ }^{5}$ Observational Cosmology Laboratory, NASA Goddard Space Flight Center, Greenbelt, MD 20771, USA \\ ${ }^{6}$ Instituto de Astrofísica, Facultad de Física, Pontificia Universidad Católica de Chile, 306, Santiago 22, Chile \\ ${ }^{7}$ Instituto de Física y Astronomía, Facultad de Ciencias, Universidad de Valparaíso, Gran Bretana 1111, Playa Ancha, Valparaíso, Chile \\ ${ }^{8}$ Millennium Institute of Astrophysics, Vicuña Mackenna 4860, 7820436 Macul, Santiago, Chile \\ ${ }^{9}$ Space Science Institute, 4750 Walnut Street, Suite 205, Boulder, CO 80301, USA \\ ${ }^{10}$ Space Sciences Laboratory, University of California, Berkeley, CA 94720, USA \\ ${ }^{11}$ DTU Space-National Space Institute, Technical University of Denmark, Elektrovej 327, DK-2800 Lyngby, Denmark \\ 12 INAF-Osservatorio Astronomico di Bologna, Via Ranzani 1, I-40127 Bologna, Italy \\ ${ }^{13}$ Lawrence Livermore National Laboratory, Livermore, CA 94550, USA \\ ${ }^{14}$ Department of Physics, Virginia Tech, Blacksburg, VA 24061, USA \\ ${ }^{15}$ Columbia Astrophysics Laboratory, Columbia University, New York, NY 10027, USA \\ ${ }^{16}$ Cahill Center for Astronomy and Astrophysics, California Institute of Technology, Pasadena, CA 91125, USA \\ ${ }^{17}$ Institute for Astronomy, Department of Physics, ETH Zurich, Wolfgang-Pauli-Strasse 27, CH-8093 Zurich, Switzerland \\ ${ }^{18}$ IPAC, California Institute of Technology, Mail Code 220-6, Pasadena, CA 91125, USA \\ ${ }^{19}$ ASDC-ASI, Via del Politecnico, I-00133 Roma, Italy \\ ${ }^{20}$ INAF-Osservatorio Astronomico di Roma, via Frascati 33, I-00040 Monte Porzio Catone (RM), Italy \\ ${ }^{21}$ Department of Astronomy, University of Maryland, College Park, MD 20742, USA \\ ${ }^{22}$ NASA Goddard Space Flight Center, Greenbelt, MD 20771, USA \\ Received 2014 May 21; accepted 2014 August 14; published 2014 September 24
}

\begin{abstract}
We report $N U S T A R$ observations of a sample of six X-ray weak broad absorption line (BAL) quasars. These targets, at $z=0.148-1.223$, are among the optically brightest and most luminous BAL quasars known at $z<1.3$. However, their rest-frame $\approx 2 \mathrm{keV}$ luminosities are 14 to $>330$ times weaker than expected for typical quasars. Our results from a pilot NUSTAR study of two low-redshift BAL quasars, a Chandra stacking analysis of a sample of highredshift BAL quasars, and a NuSTAR spectral analysis of the local BAL quasar Mrk 231 have already suggested the existence of intrinsically X-ray weak BAL quasars, i.e., quasars not emitting X-rays at the level expected from their optical/UV emission. The aim of the current program is to extend the search for such extraordinary objects. Three of the six new targets are weakly detected by NuSTAR with $\lesssim 45$ counts in the $3-24 \mathrm{keV}$ band, and the other three are not detected. The hard X-ray (8-24 keV) weakness observed by NuSTAR requires Compton-thick absorption if these objects have nominal underlying X-ray emission. However, a soft stacked effective photon index $\left(\Gamma_{\text {eff }} \approx 1.8\right)$ for this sample disfavors Compton-thick absorption in general. The uniform hard X-ray weakness observed by $N U S T A R$ for this and the pilot samples selected with $<10 \mathrm{keV}$ weakness also suggests that the X-ray weakness is intrinsic in at least some of the targets. We conclude that the NUSTAR observations have likely discovered a significant population ( $\gtrsim 33 \%$ ) of intrinsically X-ray weak objects among the BAL quasars with significantly weak $<10 \mathrm{keV}$ emission. We suggest that intrinsically X-ray weak quasars might be preferentially observed as BAL quasars.
\end{abstract}

Key words: accretion, accretion disks - galaxies: active - galaxies: nuclei - quasars: absorption lines - quasars: emission lines - X-rays: galaxies

Online-only material: color figures

\section{INTRODUCTION}

$\mathrm{X}$-ray emission is considered to be ubiquitous from active galactic nuclei (AGNs), and it is believed to originate from a hot "corona" surrounding the inner accretion disk via Comptonization of disk optical/UV/EUV photons (e.g., Haardt \& Maraschi 1991). X-ray emission may be enhanced in radio-loud AGNs due to the contribution from jets, and the X-ray weakness observed in some AGNs is generally attributed to absorption. After excluding radio-loud AGNs and potentially X-ray absorbed AGNs, a highly significant correlation between the AGN UV luminosity (2500 ̊ monochromatic luminosity, $L_{2500 \AA}$ ) and the X-ray-tooptical power-law slope parameter $\left(\alpha_{\mathrm{OX}}\right)^{23}$ has been established across $\approx 5$ orders of magnitude in UV luminosity (e.g., Steffen et al. 2006; Just et al. 2007; Lusso et al. 2010). This relation highlights apparently uniform physical mechanisms at work at the heart of the AGN engine.

\footnotetext{
${ }^{23} \alpha_{\mathrm{OX}}$ is defined as $\alpha_{\mathrm{OX}}=-0.3838 \log \left(f_{2500} \AA / f_{2 \mathrm{keV}}\right)$, where $f_{2500 \AA}$ and $f_{2 \mathrm{keV}}$ are the rest-frame $2500 \AA$ and $2 \mathrm{keV}$ flux densities.
} 
One might naturally wonder whether there are AGNs that are intrinsically X-ray weak, producing much less X-ray emission than expected from the $\alpha_{\mathrm{OX}}-L_{2500 \AA}$ relation. One such example is PHL 1811, a very bright quasar at $z=0.19$ with a $B$-band magnitude of 13.9 that has been studied extensively (e.g., Leighly et al. 2007a, 2007b). It is believed to be intrinsically $\mathrm{X}$-ray weak by a factor of $\approx 30-100$. A small sample of Sloan Digital Sky Survey (SDSS; York et al. 2000) quasars with similar emission-line properties, termed "PHL 1811 analogs," has also been observed to be X-ray weak (Wu et al. 2011), although the nature of their X-ray weakness (intrinsic X-ray weakness or absorption) is uncertain. The fraction of PHL 1811 analogs in the radio-quiet quasar population is small, $\lesssim 1.2 \%$. As a first attempt to constrain the fraction of intrinsically X-ray weak AGNs systematically, Gibson et al. (2008) searched for such objects among optically selected SDSS quasars, again excluding radio-loud AGNs and potentially X-ray absorbed systems. Their conclusion was that such AGNs are rare; e.g., the fraction is $\lesssim 2 \%$ for AGNs that are intrinsically X-ray weak by a factor of 10 or more. Discovery of intrinsically X-ray weak AGNs challenges the idea of a universal X-ray emission mechanism, and studies of such objects should provide insights into the nature of the corona.

There is one significant population of AGNs that belongs to the category of potentially X-ray absorbed AGNs which has been excluded in previous searches for intrinsically X-ray weak AGNs, and this is broad absorption line (BAL) quasars. BAL quasars comprise $\approx 15 \%$ of optically selected quasars (e.g., Hewett \& Foltz 2003; Trump et al. 2006; Gibson et al. 2009; Allen et al. 2011), and observationally they are in general X-ray weak, often due to absorption (e.g., Gallagher et al. 2002a, 2006; Fan et al. 2009; Gibson et al. 2009). In the accretion-disk wind model (see Figure 1 of Luo et al. 2013, hereafter L13), where a radiatively driven wind is launched from the accretion disk at $\approx 10^{16}-10^{17} \mathrm{~cm}$ (e.g., Murray et al. 1995; Proga et al. 2000), BALs are observed when the inclination angle is large and the line of sight passes through the outflowing wind. In this model, suppression of the nuclear X-ray emission is required to prevent the wind from being overionized. Some "shielding" material, e.g., the shielding gas as shown in Figure 1 of L13, is usually invoked to provide X-ray absorption in BAL quasars, which is consistent with the absorption typically observed. However, if a BAL quasar were intrinsically X-ray weak, the wind could also be launched successfully with little or no shielding.

Indeed, there are some BAL quasars with significant $\mathrm{X}$-ray weakness that cannot be accounted for by the apparent X-ray absorption determined using $<10 \mathrm{keV}$ Chandra or XMM-Newton data (e.g., Sabra \& Hamann 2001; L13). These are candidates for intrinsically X-ray weak AGNs. It is also possible that they are intrinsically X-ray normal but are heavily obscured $\left(N_{\mathrm{H}} \gtrsim 5 \times 10^{23} \mathrm{~cm}^{-2}\right)$ or even Compton-thick $\left(N_{\mathrm{H}} \geqslant 1.5 \times 10^{24} \mathrm{~cm}^{-2}\right)$, so that the observed $<10 \mathrm{keV}$ spectra are dominated by a Compton-reflected component. To distinguish between the intrinsic X-ray weakness and heavy absorption scenarios, observations of highly penetrating X-rays in the $>10 \mathrm{keV}$ rest-frame band are required.

In L13, we presented NuSTAR (Harrison et al. 2013) 3-79 keV observations of a pilot sample of two such BAL quasars, PG $1004+130(z=0.241)$ and PG $1700+518(z=0.292)$. Both objects are surprisingly X-ray weak in the NuSTAR band, suggesting either intrinsic X-ray weakness or highly Comptonthick absorption $\left(N_{\mathrm{H}} \approx 7 \times 10^{24} \mathrm{~cm}^{-2}\right)$. Additionally, a Chandra stacking analysis in L13 with the Large Bright Quasar Survey
(LBQS) BAL-quasar sample at high redshift (where Chandra probes the rest-frame $\approx 1.5-24 \mathrm{keV}$ band) revealed an effective power-law photon index of $\Gamma_{\text {eff }}=1.6_{-0.5}^{+0.6}$. This effective photon index is fairly soft/steep for a spectrum expected to be absorbed, and it argues against Compton-thick absorption in general, which would usually result in a hard/flat $\left(\Gamma_{\text {eff }} \approx 0.5\right.$ with a range of $\approx 0-1$ ) spectrum (e.g., George \& Fabian 1991; Comastri et al. 2011; Gandhi et al. 2014; Rovilos et al. 2014). This result suggests a significant fraction $(\approx 17-40 \%)$ of intrinsically $\mathrm{X}$-ray weak BAL quasars in this sample. Subsequently, NuSTAR observations of the nearest BAL quasar, Mrk 231, obtained hard X-ray spectra with sufficient photon statistics for spectral fitting. A joint Chandra and NuSTAR spectral analysis, though challenging due to the substantial spectral complexity present, suggests Compton-thin absorption $\left(N_{\mathrm{H}} \approx 1.2 \times 10^{23} \mathrm{~cm}^{-2}\right)$, making Mrk 231 intrinsically X-ray weak by a factor of $\approx 10$ (Teng et al. 2014). These NuSTAR and Chandra results provide the first clear evidence for the existence of intrinsically X-ray weak BAL quasars.

As an extension of the L13 pilot program, we obtained 20-35 ks NuSTAR observations of an additional six BAL quasars that show significant X-ray weakness in the $<10 \mathrm{keV}$ band. The aim is to evaluate whether they show similar hard X-ray weakness to the pilot sample. The nature of the X-ray weakness, whether intrinsic or due to Compton-thick absorption, can be assessed via stacking analyses or statistical arguments for the full sample of eight BAL quasars, including the two L13 objects. We describe the sample selection and $N u S T A R$ data analysis in Sections 2 and 3. The stacking analyses and column-density constraints are presented in Section 4. We discuss the possibility of intrinsic X-ray weakness for our sample in Section 5, and we summarize in Section 6. Throughout this paper, we use J2000.0 coordinates and a cosmology with $H_{0}=70.4 \mathrm{~km} \mathrm{~s}^{-1} \mathrm{Mpc}^{-1}$, $\Omega_{\mathrm{M}}=0.272$, and $\Omega_{\Lambda}=0.728$ (e.g., Komatsu et al. 2011). Full names of the targets are listed in the tables while abbreviated names are used in the text. We quote uncertainties at a $1 \sigma$ confidence level and upper and lower limits at a $90 \%$ confidence level, unless otherwise stated.

\section{SAMPLE SELECTION}

We selected BAL-quasar targets based on the following four criteria.

1. The targets are bona-fide BAL quasars with C IV $\lambda 1549$ absorption-trough widths $>2000 \mathrm{~km} \mathrm{~s}^{-1}$.

2 . The targets are optically bright $\left(m_{B} \lesssim 16\right)$ so that we would expect a significant number of hard X-ray photons detected with $N U S T A R$ provided they have nominal underlying $\mathrm{X}$-ray emission as expected from the $\alpha_{\mathrm{OX}}-L_{2500 \AA}$ relation and they are not Compton-thick.

3. The targets are significantly X-ray weak in the $\lesssim 10 \mathrm{keV}$ band with X-ray weakness that cannot be accounted for by any apparent X-ray absorption determined using Chandra or XMM-Newton data. ${ }^{24}$ Therefore, they are either intrinsically X-ray weak or heavily obscured. NUSTAR $>10 \mathrm{keV}$ observations will help to discriminate between these two scenarios.

\footnotetext{
24 We require a factor of $\gtrsim 10$ times $\mathrm{X}$-ray weakness at $\approx 2 \mathrm{keV}$ by comparing the measured $\alpha_{\mathrm{OX}}$ parameter to the one expected from the $\alpha_{\mathrm{OX}}-L_{2500 \AA}$ relation, and also a factor of $\gtrsim 2$ times X-ray weakness in the observed 2-8 keV band by measuring the $\alpha_{\mathrm{OX}}$,corr parameter (see Section 1.1 of L13 for discussion of $\left.\alpha_{\mathrm{OX} \text {, corr }}\right)$. In fact, the objects ultimately selected for our sample generally exceed these requirements by a substantial margin (see below).
} 

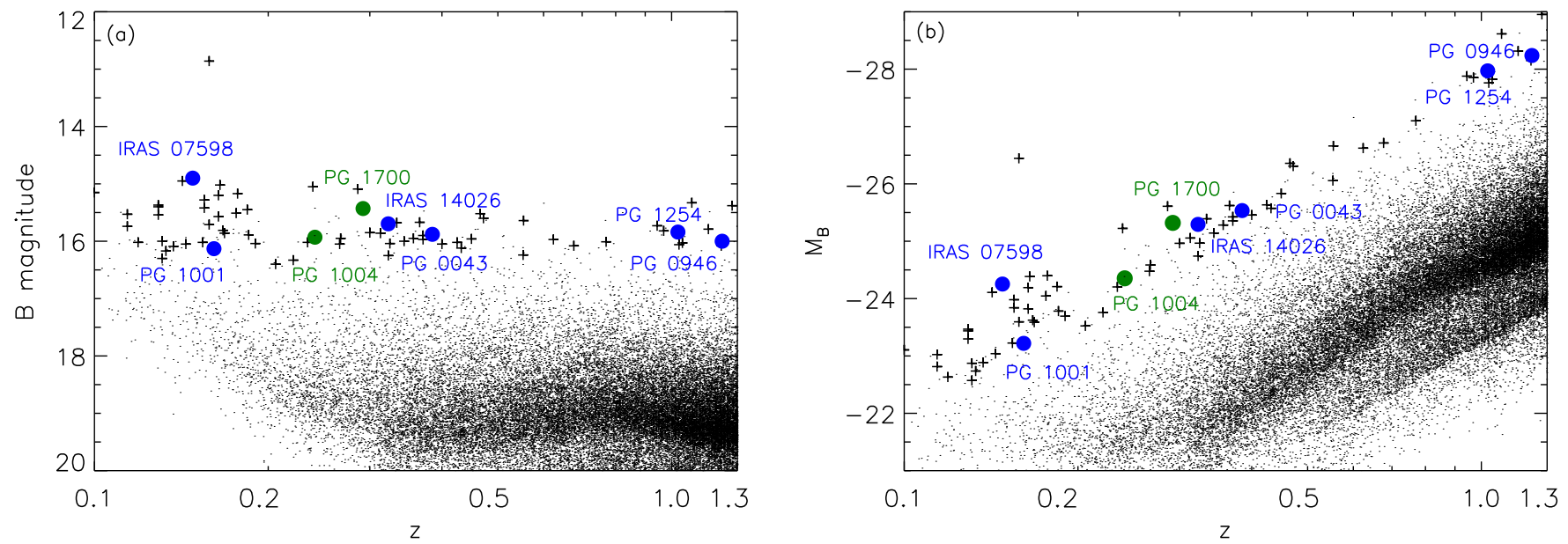

Figure 1. Redshift vs. (a) apparent and (b) absolute $B$-band magnitudes for our sample objects (blue filled circles). The green filled circles represent the two targets in L13. The plus signs are the bright PG quasars from Schmidt \& Green (1983); the brightest object is PG 1226+023 (3C 273). The underlying black dots are objects from the SDSS DR7 quasar catalog (Schneider et al. 2010). The $B$-band magnitudes of the SDSS quasars were converted from the $g$-band magnitudes, assuming an optical power-law slope of $\alpha_{0}=-0.5\left(f_{v} \propto v^{\alpha}\right.$; e.g., Vanden Berk et al. 2001). The $K$-corrections were performed assuming the same optical power-law slope. Our targets are among the optically brightest and most luminous BAL quasars known at $z<1.3$.

(A color version of this figure is available in the online journal.)

Table 1

NuSTAR Observation Log and Target Properties

\begin{tabular}{|c|c|c|c|c|c|c|c|c|c|c|c|}
\hline $\begin{array}{l}\text { Object } \\
\text { Name } \\
\text { (1) }\end{array}$ & (2) & $m_{B}$ & $M_{B}$ & $\begin{array}{c}\text { Observation } \\
\text { Start Date } \\
\text { (5) }\end{array}$ & $\begin{array}{c}\text { Observation } \\
\text { ID } \\
\text { (6) }\end{array}$ & $\begin{array}{l}\operatorname{Exp} \\
(\mathrm{ks}) \\
(7)\end{array}$ & $\begin{array}{c}\text { Exp_clean } \\
\text { (ks) } \\
(8)\end{array}$ & $\begin{array}{c}\Delta_{\mathrm{OX}} \\
(\operatorname{arcsec}) \\
(9)\end{array}$ & $\begin{array}{c}\log M_{\mathrm{BH}} \\
\left(M_{\odot}\right) \\
(10)\end{array}$ & $\begin{array}{c}\log L_{\text {bol }} \\
\left(\mathrm{erg} \mathrm{s}^{-1}\right) \\
(11)\end{array}$ & $\begin{array}{l}\text { BAL } \\
\text { Type } \\
(12)\end{array}$ \\
\hline PG 0043+039 & 0.385 & 15.9 & -25.5 & $2013 \mathrm{Jul} 18$ & 60001119002 & 21.5 & 20.2 & . & $9.0^{\mathrm{a}}$ & 46.2 & HiBAL \\
\hline IRAS $07598+6508$ & 0.148 & 14.9 & -24.3 & 2013 Oct 29 & 60001120002 & 30.2 & 28.1 & .. & $8.3^{\mathrm{a}}$ & 46.2 & LoBAL \\
\hline PG 0946+301 & 1.223 & 16.0 & -28.2 & 2013 Nov 09 & 60001124002 & 37.4 & 34.9 & 6.9 & $9.8^{\mathrm{b}}$ & 47.5 & HiBAL \\
\hline PG $1001+054$ & 0.161 & 16.1 & -23.2 & 2013 Jun 28 & 60001122002 & 20.9 & 19.6 & 8.0 & $7.7^{\mathrm{b}}$ & 45.5 & HiBAL \\
\hline PG 1254+047 & 1.026 & 15.8 & -28.0 & 2013 Jun 27 & 60001123002 & 31.7 & 29.4 & 2.9 & $9.7^{\mathrm{b}}$ & 47.3 & HiBAL \\
\hline IRAS $14026+4341$ & 0.323 & 15.7 & -25.3 & 2013 Nov 10 & 60001121002 & 27.8 & 26.0 & $\ldots$ & $8.6^{\mathrm{b}}$ & 46.3 & LoBAL \\
\hline
\end{tabular}

Notes. Columns 1 and 2: object name and redshift; Columns 3 and 4: apparent and absolute $B$-band magnitudes; Columns 5 and 6: NuSTAR observation start date and observation ID; Columns 7 and 8: nominal and cleaned NuSTAR exposure times, respectively; Column 9: minimum positional offset between the optical and X-ray positions. The X-ray positions are determined from waVDETECT detections in the 3-24 keV images of FPMA and FPMB. Blank entries indicate non-detections; Column 10: virial BH mass from the literature; (a) Hao et al. (2005) and (b) Shen et al. (2011); Column 11: bolometric luminosity calculated from the scaled Richards et al. (2006) composite quasar SED. Column 12: BAL quasar type depending upon whether there are BALs from ions at low-ionization states.

4. The targets are radio quiet (radio-loudness parameter $R<10)^{25}$ so that their X-ray emission is not contaminated by any jet-linked emission.

We searched for such targets in the $z<0.5$ Palomar-Green (PG) quasar sample (Schmidt \& Green 1983) and in literature reports of BAL quasars with significant X-ray weakness. The six targets are listed in Table 1, of which PG 0043 and PG 1001 are among the $z<0.5$ PG quasar sample, while IRAS 07598 (e.g., Gallagher et al. 1999; Imanishi \& Terashima 2004; Saez et al. 2012), PG 0946 (e.g., Mathur et al. 2000; Saez et al. 2012), PG 1254 (e.g., Sabra \& Hamann 2001), and IRAS 14026 (e.g., Saez et al. 2012) are from the literature. These BAL quasar targets have $B$-band magnitudes of $\approx 15-16$, and they are among the optically brightest and most luminous BAL quasars known at $z<1.3$ (Figure 1). The more luminous PG 0946 and PG 1254 are also representative counterparts of the luminous BAL quasars typically studied at $z \approx 2-3$ (e.g., Gibson et al. 2009).

${ }^{25} R=f_{5 \mathrm{GHz}} / f_{4400 \AA}$ (the ratio between the $5 \mathrm{GHz}$ and $4400 \AA$ Alux densities in the rest frame; e.g., Kellermann et al. 1989). We obtained radio flux information from the Faint Images of the Radio Sky at Twenty-Centimeters (FIRST) survey (Becker et al. 1995) or the NRAO VLA Sky Survey (NVSS; Condon et al. 1998).
There are five BAL quasars among the $z<0.5$ PG quasar sample (see Footnote 4 of Brandt et al. 2000), four of which have now been included in our NUSTAR BAL quasar program (PG 0043 and PG 1001 here and PG 1004 and PG 1700 in L13). ${ }^{26}$ Therefore, we are sampling a significant fraction of the most luminous BAL quasars at low redshifts. The sample completeness among the general population of BAL quasars is not clear as there have been no systematic identifications of BAL quasars at low redshifts, which usually require Hubble Space Telescope spectra covering the key C IV $\lambda 1549$ transition.

All six targets have archival Chandra and/or XMM-Newton observations. The X-ray observations of IRAS 07598, PG 0946, PG 1001, and IRAS 14026 are summarized in Saez et al. (2012) and references therein. PG 1254 has a 36 ks Chandra observation that was reported in Sabra \& Hamann (2001). PG 0043 has two $\approx 30 \mathrm{ks} X M M-N e w t o n$ observations. The source was not detected in the first observation and data for the second observation are not publicly available yet. We reprocessed the public Chandra and XMM-Newton data and obtained the $\alpha_{\mathrm{OX}}$ parameters for the targets, with $f_{2 \mathrm{keV}}$ derived

\footnotetext{
26 The other BAL quasar is PG $2112+059$, the X-ray weakness of which can
} be explained by moderate absorption (e.g., Gallagher et al. 2001, 2004). 


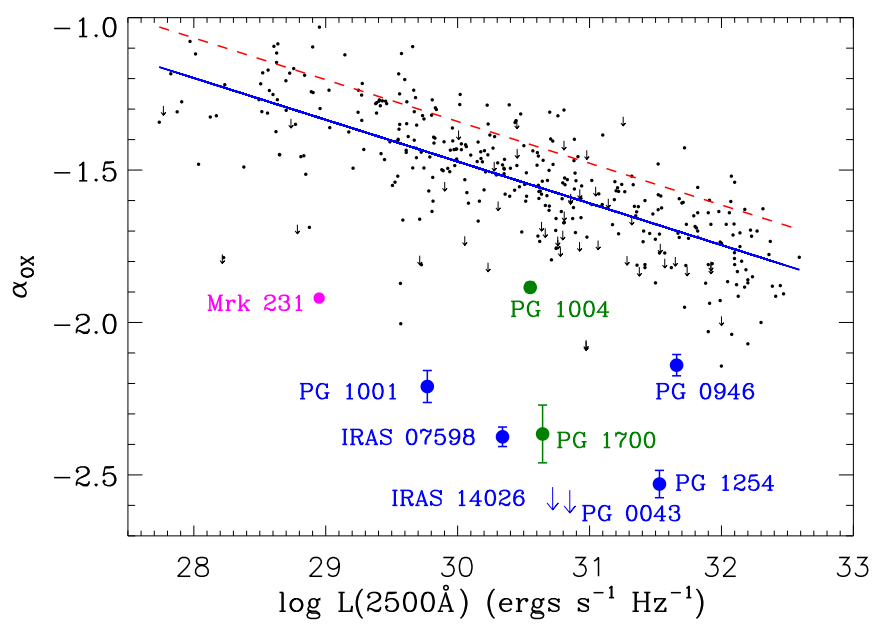

Figure 2. X-ray-to-optical power-law slope $\left(\alpha_{\mathrm{OX}}\right)$ vs. $2500 \AA$ monochromatic luminosity (not corrected for intrinsic reddening) for the six targeted X-ray weak BAL quasars (blue data points). Also shown are the two objects in the pilot sample (green; L13) and Mrk 231 (magenta; Teng et al. 2014). The small black dots and downward arrows (upper limits) are from the sample of Steffen et al. (2006) with the solid blue line showing the $\alpha_{\mathrm{OX}}-L_{2500 \AA}$ relation. The dashed red line represents the Steffen et al. (2006) relation modified with the excess X-ray luminosity expected for the radio loudness of PG 1004+130 (Miller et al. 2011). All these BAL quasars are significantly X-ray weak at rest-frame $\approx 2 \mathrm{keV}$.

(A color version of this figure is available in the online journal.)

from the observed $0.5-2 \mathrm{keV}$ flux and $f_{2500 \AA}$ derived by interpolating the optical-UV photometric data (see Section 5.1 below). The $\alpha_{\mathrm{OX}}$ constraints for the targets are shown in Figure 2, which indicate that their rest-frame $\approx 2 \mathrm{keV}$ luminosities are 14 to $>330$ times weaker than expected from the Steffen et al. (2006) $\alpha_{\mathrm{OX}}-L_{2500 \AA}$ relation. These $\alpha_{\mathrm{OX}}$ values have not been corrected for any intrinsic optical/UV reddening, which would render the values even more negative. Note that the spread of the Steffen et al. (2006) data points constrains the expected amount of quasar variability (e.g., Gibson \& Brandt 2012), and after accounting for the measured flux variability of our targets (see Section 3 below), they are still significantly X-ray weak. Also evident in Figure 2 is the significant $\approx 2 \mathrm{keV}$ weakness of PG 1004, PG 1700, and Mrk 231 (L13; Teng et al. 2014). In addition, we verified that these targets are still X-ray weak (by factors of $\approx 2$ to $>220$ ) in a somewhat harder band by measuring $\alpha_{\mathrm{OX} \text {, corr }}$ with the observed $2-8 \mathrm{keV}$ flux assuming a $\Gamma=1.8$ power-law spectrum (see Section 1.1 of L13). Given the significant X-ray weakness of these six targets, they could be either heavily obscured or intrinsically X-ray weak.

There have been no previous tight constraints on the $>10 \mathrm{keV}$ emission of these objects. They are not detected in the 70 month Swift-BAT 14-195 keV all-sky survey (Baumgartner et al. 2013), the sensitivity of which falls short by an order of magnitude even if they have nominal hard X-ray emission. With the much higher sensitivity of NuSTAR, the hard X-ray emission of these objects can be sensitively constrained with relatively short exposure times. Assuming that the targets are intrinsically X-ray normal with an underlying $2 \mathrm{keV}$ luminosity determined by the $\alpha_{\mathrm{OX}}-L_{2500 \AA}$ relation, we can estimate the NUSTAR counts yield following the approach in Section 4.1.1 of L13 using the MYTorus model (Murphy \& Yaqoob 2009). Provided that the sources are Compton-thin $\left(N_{\mathrm{H}}<1.5 \times\right.$ $10^{24} \mathrm{~cm}^{-2}$ ), we would expect significant detections of PG 0043 , IRAS 07598, PG 1001, and IRAS 14026 in the 8-24 keV band (more than 80-260 counts where these values are derived for
$N_{\mathrm{H}}=1.5 \times 10^{24} \mathrm{~cm}^{-2}$ ) with $20 \mathrm{ks}$ NUSTAR observations. Longer exposures are required for the more distant targets PG 0946 (35 ks) and PG 1254 (30 ks) for a 8-24 keV detection with more than 30 counts. Fewer counts are expected, of course, if the targets are either intrinsically X-ray weak or have Compton-thick column densities substantially exceeding $1.5 \times 10^{24} \mathrm{~cm}^{-2}$.

\section{NuSTAR OBSERVATIONS AND DATA ANALYSIS}

The details of the NUSTAR observations are listed in Table 1 . We processed the level 1 data using the NuSTAR Data Analysis Software (NuSTARDAS) v.1.2.0 with NuSTAR CALDB 20131007, and produced cleaned calibrated event files (level 2 data) using the NUPIPELINE script for both focal plane modules (FPMs, including FPMA and FPMB; Harrison et al. 2013). For each target in each FPM, we created X-ray images in four bands: 3-24 keV, 3-8 keV, 8-24 keV, and 24-79 keV using the Chandra Interactive Analysis of Observations (CIAO) ${ }^{27}$ v4.5 tool DMCOPY. These bands are being adopted as standard photometric bands in current NuSTAR studies (e.g., Alexander et al. 2013; Lansbury et al. 2014), but they are slightly different from those used in the early L13 pilot study (e.g., 4-20 keV, 4-10 keV, and 10-20 keV bands). We have verified that alternative choices of photometric bands yield consistent results.

We searched for sources in each of the images using the CIAO tool WAVDETECT (Freeman et al. 2002) with a false-positive probability threshold of $10^{-5}$ and wavelet scales of $2,2.83,4$, $5.66,8,11.31$, and 16 pixels (the pixel size is 2 .'46). PG 0946, PG 1001, and PG 1254 are detected in at least one band in each FPM. The minimum positional offsets (Table 1) between the optical and 3-24 keV positions are within expectations for faint sources. The chance of getting any spurious detections by WAVDETECT at these known source positions is negligible. The other three targets, PG 0043, IRAS 07598, and IRAS 14026, are not detected; we also verified the non-detections via visual inspection of the smoothed images. None of the targets is detected in the $24-79 \mathrm{keV}$ band, and the constraints from the non-detections in this band are not as tight as those in the other bands. Stacking in this band does not yield any useful constraints either. Therefore, we do not include the $24-79 \mathrm{keV}$ band in the following discussion.

We performed aperture photometry for each target in the three standard bands above. Total (source plus background) counts were extracted within a $35^{\prime \prime}$-radius circular aperture, centered on the 3-24 keV position (for detected targets) or optical position (for undetected targets). This aperture approximates the $63.9 \%$ encircled-energy fraction contour of the point spread function, and we have verified that different choices of the source-extraction region yield consistent results. Background counts were extracted from a simulated background map created using the NUSKYBGD script (Wik et al. 2014); these are consistent with those estimated from annular or circular off-source regions. We followed the binomial no-source probability $\left(P_{\mathrm{B}}\right)$ approach in L13 to determine the source detection significance in each $N u S T A R$ band. If the $P_{\mathrm{B}}$ value is smaller than $0.01(\approx 2.6 \sigma)$, we considered the source detected and calculated the $1 \sigma$ errors on the net counts (Gehrels 1986). If the $P_{\mathrm{B}}$ value is larger than 0.01 , we considered the source undetected and derived an upper limit on the source counts using the Bayesian approach of Kraft et al. (1991). The aperture-corrected source counts and upper limits for our targets are listed in Table 2. Measurements for

\footnotetext{
27 See http://cxc.harvard.edu/ciao/ for details on CIAO.
} 
Table 2

NUSTAR Photometry and Column-density Constraints

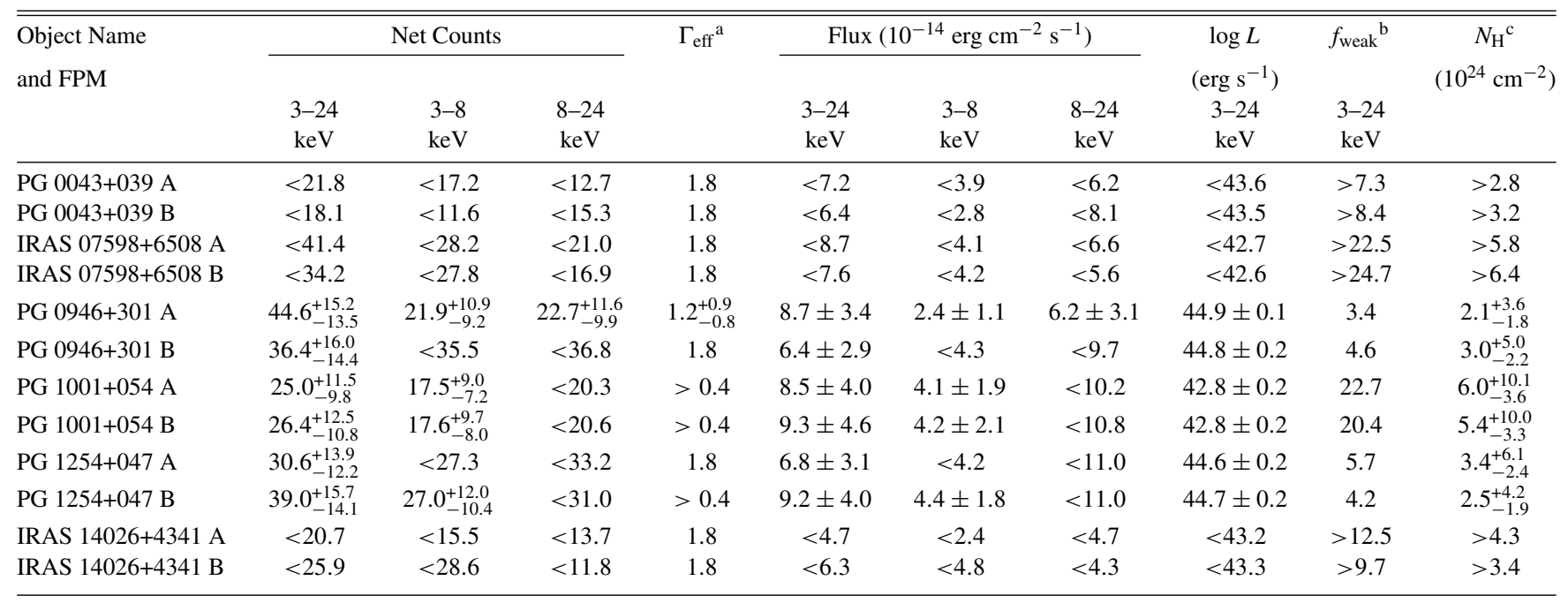

Notes.

${ }^{\text {a }}$ Effective photon index, derived based on the band ratio between the observed 8-24 keV and 3-8 keV counts, assuming a power-law model with Galactic absorption. $\Gamma_{\text {eff }}=1.8$ is assumed if it cannot be constrained.

${ }^{\mathrm{b}}$ Factor of X-ray weakness in the $3-24 \mathrm{keV}$ band, $f_{\text {weak }}=F_{\text {expected }} / F_{\text {observed }}$. The expected flux was derived based on the expected $\alpha_{\mathrm{OX}}\left(\right.$ from the $\alpha_{\mathrm{OX}}-L_{2500} \AA$ relation) and a power-law X-ray spectrum with $\Gamma=1.8$. The scatter of the expected flux was accounted for when deriving the lower limit for an undetected source.

c Absorption column-density constraint derived from the factor of 3-24 keV weakness using the MYToRUS model.

FPMA and FPMB are consistent within the uncertainties. In the 8-24 keV band, none of the targets is detected except PG 0946 in FPMA with a 23 count detection; the source counts are below our expectations for the Compton-thin scenario (Section 2), and thus all targets are hard X-ray weak, similar to the pilot sample in L13.

Since a relatively large aperture with a $35^{\prime \prime}$ radius was used in photometry extraction, we investigated whether there are any contaminating sources nearby. We inspected the Chandra or XMM-Newton images for our targets, and confirmed that there is no neighboring source within $50^{\prime \prime}$ of the targets except PG 0043. There is one source 25" away from PG 0043 that was reported in Ballo et al. (2008). The XMM-Newton data show a soft spectrum $(\Gamma=1.86)$ with a $2-10 \mathrm{keV}$ flux of $1.8 \times$ $10^{-14} \mathrm{erg} \mathrm{cm}^{-2} \mathrm{~s}^{-1}$. We estimated that the contamination from this source in our aperture is negligible $(\approx 0.8$ counts in the $3-8 \mathrm{keV}$ band and $\approx 0.5$ counts in the $8-24 \mathrm{keV}$ band). This source should not affect our stacking results below (Section 4.1) either.

Following L13, we derived a 3-24 keV effective power-law photon index $\left(\Gamma_{\text {eff }}\right)$ from the band ratio between the $8-24 \mathrm{keV}$ and $3-8 \mathrm{keV}$ bands, calibrated using the NuSTAR spectral response files extracted at the source location and assuming a power-law spectrum with the Galactic absorption column density (Dickey \& Lockman 1990). The uncertainties of (or limits on) the band ratios (and subsequently $\Gamma_{\text {eff }}$ ) were derived using the Bayesian code BEHR (Park et al. 2006). For sources undetected in both the $8-24 \mathrm{keV}$ and $3-8 \mathrm{keV}$ bands, $\Gamma_{\text {eff }}=1.8$ was adopted. The $\Gamma_{\text {eff }}$ values are listed in Table 2, which do not individually provide tight constraints on whether the sources have hard (indicative of absorption) or soft spectra, due to the non-detections or large uncertainties. The source fluxes and luminosities, listed in Table 2, were converted from the count rates and $\Gamma_{\text {eff }}$, calibrated with the spectral response files.

We compared the NUSTAR 3-8 keV flux measurements to previous Chandra and XMM-Newton data. Long-term flux variability is observed in the three detected targets. The $3-8 \mathrm{keV}$ flux of PG 0946 has dropped by a factor of $5.4 \pm 2.8$ between the 2010 Chandra observation (Saez et al. 2012) and the NuSTAR observation. The NuSTAR flux of PG 1001 is consistent with that measured in the 2003 XMM-Newton observation, and it is $4.9 \pm 2.9$ times higher than the flux in the 2010 Chandra observation (Saez et al. 2012). PG 1254 is $2.7 \pm 1.4$ times brighter in the NUSTAR observation compared to the 2000 Chandra observation (Sabra \& Hamann 2001). We note that after accounting for their flux increases, PG 1001 and PG 1254 are still 21 and 58 times X-ray weak at $\approx 2 \mathrm{keV}$ (e.g., in Figure 2), respectively. Similar flux variability has also been noted in PG 1004 in the pilot sample (Miller et al. 2006; L13) and several other BAL quasars (e.g., Gallagher et al. 2004; Saez et al. 2012). For the other three undetected targets, the flux upper limits on IRAS 07598 and IRAS 14026 are consistent with previous Chandra and/or XMM-Newton flux measurements, and PG 0043 is not detected by XMM-Newton either. For IRAS 07598 and IRAS 14026, combining the 3-8 keV fluxes from Chandra or XMM-Newton and the 8-24 keV flux upper limits from $N u S T A R$ does not provide useful constraints on $\Gamma_{\text {eff }}$. It is not useful to combine the lower energy data with the NuSTAR data for the three detected targets due to the observed variability.

\section{RESULTS}

\subsection{Stacking and Joint Spectral Analyses}

Since our targets are only weakly detected or undetected by $N u S T A R$, we cannot study the nature of their hard X-ray weakness via individual spectral analysis. Instead, we performed stacking and joint spectral analyses to probe the average spectral properties of the sample. First, we stacked the FPMA and FPMB data for each object individually. The three undetected targets are still not detected. PG 0946 and PG 1254 are detected in the 8-24 keV band, allowing better constraints on their effective photon indices. For PG 0946, we obtained a $\Gamma_{\text {eff }}$ of $1.2_{-0.6}^{+0.7}$, 
consistent with its FPMA measurement. For PG $1254, \Gamma_{\text {eff }}$ is $1.5_{-0.6}^{+0.8}$.

Stacking of the full sample of six objects in both FPMA and FPMB yields significant detections in both the $3-8 \mathrm{keV}$ and $8-24 \mathrm{keV}$ bands, with $164.5_{-29.4}^{+31.0}$ and $102.3_{-28.3}^{+29.9}$ counts, respectively. The band ratio to $\Gamma_{\text {eff }}$ conversion factors vary slightly between different sources, and thus we adopted the average value to convert the $8-24 \mathrm{keV}$ to $3-8 \mathrm{keV}$ band ratio of the stacked source to an effective photon index, which is $\Gamma_{\text {eff }}=1.8_{-0.4}^{+0.5}$. This $\Gamma_{\text {eff }}$ represents the weighted average of the individual effective photon indices; the weight varies (by factors of a few) between sources due to their different fluxes, exposure times, and rest-frame bands probed. For the subsample of the three detected targets, the stacked counts in the $3-8 \mathrm{keV}$ and $8-24 \mathrm{keV}$ bands are $115.7_{-21.8}^{+23.5}$ and $92.0_{-22.1}^{+23.7}$, respectively, and the effective photon index is $\Gamma_{\text {eff }}=1.5_{-0.4}^{+0.4}$; for the undetected subsample, the stacked source is only weakly detected in the 3-8 keV band, and the stacked counts in the 3-8 keV and $8-24 \mathrm{keV}$ bands are $48.8_{-19.7}^{+21.3}$ and $<38.1$, respectively, with $\Gamma_{\text {eff }}>0.8$. Given the $\Gamma_{\text {eff }}$ values for the stacking of the full sample and the detected subsample, the stacked source for the undetected subsample is likely soft, e.g., the lower limit on $\Gamma_{\text {eff }}$ at a less conservative $1 \sigma$ confidence level is $\Gamma_{\text {eff }}>1.3$.

The stacked 3-8 keV counts should have negligible contribution from host-galaxy X-ray emission; the contribution is only $\approx 3 \%$ for host galaxies with a high $\mathrm{X}$-ray luminosity of $10^{42} \mathrm{erg} \mathrm{s}^{-1}$, which corresponds to a star-formation rate of $\approx 620 M_{\odot} \mathrm{yr}^{-1}$ (e.g., Lehmer et al. 2010). Therefore, the stacked signals are dominated by the nuclear sources. The soft stacked effective photon indices for the full sample $\left(1.8_{-0.4}^{+0.5}\right)$ and the detected subsample $\left(1.5_{-0.4}^{+0.4}\right)$ suggest that the targets on average are not absorbed by Compton-thick material, which would generally result in a flat $\left(\Gamma_{\text {eff }} \approx 0.5\right.$ with a range of $\left.\approx 0-1\right)$ spectrum from 3-24 keV. Moreover, the soft stacked signal for the full sample cannot be dominated by one single object given their individual count contributions in the 3-8 keV band (three sources are not detected and the other three are weakly detected), indicating that at least two objects are responsible for the soft stacked $\Gamma_{\text {eff }}$. Therefore, at least 33\% of the sample objects likely have soft effective photon indices $\left(\Gamma_{\text {eff }} \gtrsim 1.8\right)$ and are likely not Compton-thick.

We also jointly fitted the NUSTAR spectra of the three detected targets, PG 0946, PG 1001, and PG 1254, with XSPEC v.12.8.1g (Arnaud 1996). We used the NUPRODUCTS script of NuSTARDAS to extract source spectra within the same $35^{\prime \prime}$-radius circular apertures as in the photometry extraction, and local background spectra within annular regions with inner and outer radii of $120^{\prime \prime}$ and $240^{\prime \prime}$, respectively. The 3-24 keV spectra for the three targets in both FPMA and FPMB were fitted jointly with a simple power-law model using the $C$ statistic (cstat) in XSPEC, ${ }^{28}$ allowing each target to have its own redshift and Galactic column density. The best-fit photon index is $\Gamma=1.55_{-0.29}^{+0.30}(C=290$ for 335 degrees of freedom $)$, consistent with the stacked effective photon index for this subsample. The limited photon statistics of the spectra do not allow for any useful constraint on $\mathrm{Fe} \mathrm{K} \alpha$ line emission at rest-frame $6.4-6.97 \mathrm{keV}$; a strong $\mathrm{Fe} \mathrm{K} \alpha$ line with an equivalent width of the order of $1-2 \mathrm{keV}$ might be expected if the continuum is reflection dominated (e.g., Ghisellini et al. 1994; Matt et al. 1996; Gandhi

\footnotetext{
28 The $W$ statistic was actually used in the presence of background spectra; see http://heasarc.gsfc.nasa.gov/docs/xanadu/xspec/manual/XSappendixStatistics. html.
}

et al. 2014). Previous Chandra or XMM-Newton observations do not provide useful constraints on the Fe $\mathrm{K} \alpha$ line either (e.g., Imanishi \& Terashima 2004).

\subsection{Indirect Absorption Column-density Constraints}

We adopted the same approach described in Section 4.1.1 of L13 to constrain the absorption column densities indirectly for the six targets, under the assumption that the observed hard X-ray weakness is attributed entirely to absorption and they have nominal underlying X-ray emission as determined from the $\alpha_{\mathrm{OX}}-L_{2500 \AA}$ relation. Briefly, an absorption column density was derived by comparing the observed flux to the expected one derived from the expected $\alpha_{\mathrm{OX}}$ assuming a powerlaw X-ray spectrum with $\Gamma=1.8$. The MYTorus XSPEC model, including both the transmitted and scattered spectral components, was used to calibrate the relation between $N_{\mathrm{H}}$ and this X-ray weakness. ${ }^{29}$ We assumed a half-opening angle of $60^{\circ}$ (corresponding to a torus covering factor of 0.5 ) and an inclination angle of $80^{\circ}$ in the MYToRUs model. The $N_{\mathrm{H}}$ dependence on the assumed half-opening angle is relatively small, as illustrated in Figures 7 and 8 of L13 $(\approx 20 \%$ smaller for a half-opening angle of $37^{\circ}$ ). Large inclination angles are generally expected for BAL quasars in the disk-wind scenario; for inclination angles smaller than $80^{\circ}$, we would derive larger $N_{\mathrm{H}}$ values by factors of up to $\approx 3$ (Figures 7 and 8 of L13).

We derived column-density constraints using the $3-24 \mathrm{keV}$, 3-8 keV, and 8-24 keV NuSTAR fluxes or flux upper limits. The constraints in the $3-24 \mathrm{keV}$ and $8-24 \mathrm{keV}$ bands are comparable, and they are significantly tighter than those in the softer $3-8 \mathrm{keV}$ band, as one would expect given the higher rest-frame energies utilized (also see Figures 7 and 8 of L13). We list in Table 2 the factors of 3-24 keV weakness and the $3-24 \mathrm{keV} N_{\mathrm{H}}$ constraints. The $N_{\mathrm{H}}$ uncertainty accounts for the measured flux uncertainty and the spread of the intrinsic $\alpha_{\mathrm{OX}}$ value which was assumed to follow a Gaussian distribution with its $1 \sigma$ uncertainty from Table 5 of Steffen et al. (2006). For the undetected targets, the lower limits on $N_{\mathrm{H}}$ were obtained from $N_{\mathrm{H}}$ probability distributions that were derived from the $\alpha_{\mathrm{OX}}$ Gaussian distributions and the probability distributions of the $3-24 \mathrm{keV}$ fluxes. ${ }^{30}$ The column density constraints indicate that Compton-thick absorption is required for all six targets to produce the observed hard $\mathrm{X}$-ray weakness if they have nominal intrinsic X-ray emission, as expected from our experimental design.

We note that these column density constraints were derived from the observed hard X-ray weakness, independent of the spectral-shape constraints above. In fact, Compton-thick absorption is likely inconsistent with a soft $\Gamma_{\text {eff }}$, as discussed in more detail below (Section 5.2).

\section{DISCUSSION}

\subsection{Multiwavelength Properties}

As in L13, we constructed infrared (IR) to X-ray spectral energy distributions (SEDs) for the targets, using photometric data from the Wide-field Infrared Survey Explorer (WISE;

\footnotetext{
29 The physical properties of the shielding gas in BAL quasars are poorly understood (e.g., Proga \& Kallman 2004). The parameterization of the MYToRUs model cannot fully reproduce the complex absorption environments of our targets, but we consider it the best available approximation for the purpose of deriving basic column-density constraints. 30 The probability distributions of the $3-24 \mathrm{keV}$ fluxes are from the probability distributions of the $3-24 \mathrm{keV}$ counts, which were derived during our computation of the band ratios using the BEHR code.
} 

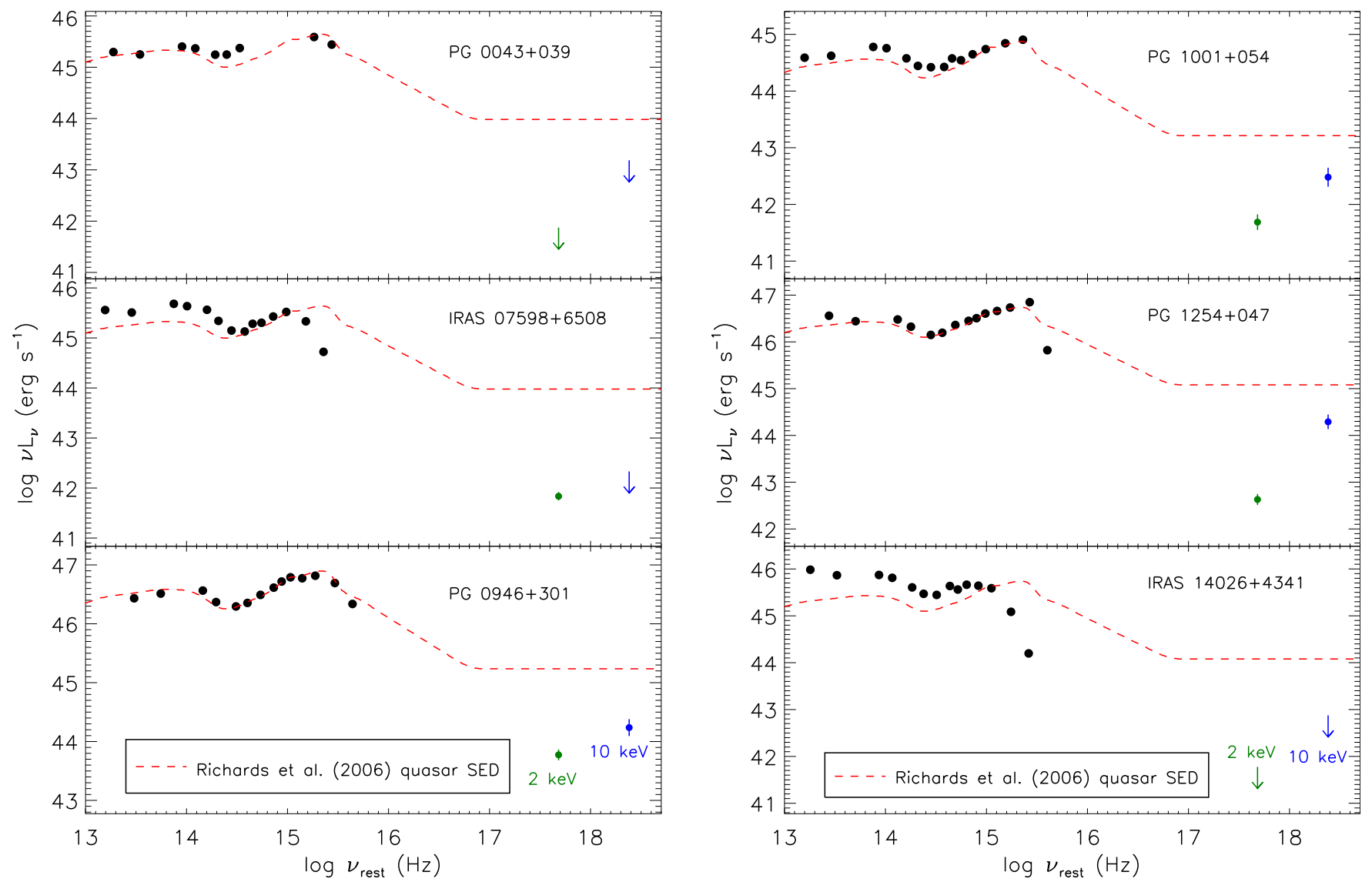

Figure 3. IR through X-ray SEDs of the six targets in the rest frame. The IR-UV data (black points) are from the WISE, 2MASS, SDSS, and/or GALEX catalogs. The $2 \mathrm{keV}$ data (green points and arrows) are from previous Chandra or XMM-Newton observations; note that IRAS 14026 was detected in the $2-8 \mathrm{keV}$ band by Chandra, but not in the $0.5-2 \mathrm{keV}$ band. The $10 \mathrm{keV}$ data (blue points and arrows) were derived from the NuSTAR 3-24 keV fluxes or flux upper limits averaged over FPMA and FPMB assuming $\Gamma_{\text {eff }}=1.2$ for PG 0946 and $\Gamma_{\text {eff }}=1.8$ for the rest of the objects. The error bars for most of the data points are smaller than or comparable to the symbol size and are thus not visible. The SED data were not observed simultaneously and may be affected by variability (e.g., see the X-ray variability in Section 3 ). The red dashed curve shows the composite quasar SED of Richards et al. (2006) normalized to the 3000 A luminosity.

(A color version of this figure is available in the online journal.)

Wright et al. 2010), Two Micron All Sky Survey (2MASS; Skrutskie et al. 2006), SDSS, and/or Galaxy Evolution Explorer (GALEX; Martin et al. 2005) catalogs. The rest-frame SEDs are shown in Figure 3. The optical and UV data have been corrected for Galactic extinction following the dereddening approach of Cardelli et al. (1989) and O'Donnell (1994). Besides the strong intrinsic reddening in IRAS 07598 and IRAS 14026 (also see Jiang et al. 2013 for the reddening in IRAS 14026) and the significant X-ray weakness, these targets have typical radio-quiet quasar SEDs (e.g., Richards et al. 2006), similar to PG 1700 in the pilot sample.

Depending upon whether there are BALs from ions at lowionization states such as $\mathrm{Mg}$ II or $\mathrm{Al}$ III, BAL quasars are classified as low-ionization BAL (LoBAL) or high-ionization BAL (HiBAL) quasars (e.g., Weymann et al. 1991; Sprayberry $\&$ Foltz 1992). LoBAL quasars constitute a minority $(\approx 10 \%)$ of BAL quasars, and they often show signs of dust reddening and are X-ray weaker than HiBAL quasars (e.g., Green et al. 2001; Gallagher et al. 2006; Gibson et al. 2009). Among our six targets here, IRAS 07598 and IRAS 14026 are LoBAL quasars (e.g., Hines \& Wills 1995; Hines et al. 2001), while the other objects are HiBAL quasars (e.g., Turnshek et al. 1994; Arav et al. 2001). The two LoBAL quasars indeed show significant dust reddening and soft and hard X-ray weakness (Figure 3 and Table 2).
It has been suggested that significant X-ray weakness may be associated with super-Eddington accretion (e.g., Lusso et al. 2010). PG 1004 and PG 1700 in the pilot sample appear to have sub-Eddington accretion (Eddington ratios $\approx 0.09$ and $\approx 0.41$; L13), while Mrk 231 indeed appears to be a super-Eddington source with an Eddington ratio of $\approx 5$ (Teng et al. 2014). We estimated bolometric luminosities for our targets from the Richards et al. (2006) composite quasar SED normalized to their $3000 \AA$ A luminosities (not strongly affected by intrinsic reddening), and collected their single-epoch virial black hole (BH) masses from the literature (Hao et al. 2005; Shen et al. 2011). These data are listed in Table 1. The derived Eddington ratios are in the range of 0.13 to 0.62 , all in the sub-Eddington regime. However, there are significant uncertainties associated with the estimated BH masses ( $>0.3$ dex; e.g., Shen \& Liu 2012) and bolometric luminosities, and thus it is difficult to assess whether the significant X-ray weakness is related to super-Eddington accretion in these cases.

The intrinsically X-ray weak quasar PHL 1811 has unusually weak and blueshifted high-ionization lines (e.g., the equivalent width of its C IV $\lambda 1549$ emission line is $6.6 \AA$, much smaller than the average value of $\approx 30 \AA$ for SDSS quasars; Leighly et al. 2007a), which may be due to the lack of high-energy ionizing continuum photons. A recent study of IRAS 14026 (Jiang et al. 2013) suggests that it is probably a PHL 1811 analog given 
the weak C IV and C III] line emission, although no quantitative measurement of the line strength has been given. Wu et al. (2011) proposed a simple unification model (e.g., Figure 9 of Wu et al. 2011) where PHL 1811 analogs and BAL quasars have similar inner structures but the lines of sight to PHL 1811 analogs do not intercept the UV-absorbing disk wind. Confirmation of such a connection would facilitate our understanding of the nature of their X-ray weakness. We visually examined the UV spectra for the other targets, the pilot sample, and also Mrk 231 (e.g., Hines \& Wills 1995; Hamann 1998; Arav et al. 1999; Brandt et al. 2000; Gallagher et al. 2002b). The C IV emission lines appear strong in IRAS 07598, PG 0946, PG 1001, PG 1254, and Mrk 231, while they are either weak or are contaminated by the broad absorption features in PG 0043, PG 1004, and PG 1700. Therefore, more than half of our targets do not show weak C IV line emission as in PHL 1811. It is possible that intrinsically $\mathrm{X}$-ray weak quasars may exhibit normal optical-UV emission lines if the accretion still produces sufficient EUV (perhaps $\lesssim 0.5 \mathrm{keV}$ ) radiation to ionize the broad line regions, e.g., photons with energies exceeding $48 \mathrm{eV}$ are required to ionize $\mathrm{C}$ III and produce the $\mathrm{C}$ IV emission line. A connection between BAL quasars and PHL 1811 analogs thus cannot be excluded.

\subsection{Intrinsic X-Ray Weakness in the Sample}

As discussed extensively in L13, the hard X-ray weakness of these BAL quasars observed by NUSTAR can, in principle, be explained in either the Compton-thick absorption or intrinsic $\mathrm{X}$-ray weakness scenarios. Without high photon counts, it is not feasible to constrain their nature individually via spectral analyses as was done for the case of the local object Mrk 231. However, we have already established that there is apparently a population of intrinsically X-ray weak BAL quasars (L13; Teng et al. 2014), and we present below some evidence that at least some of our targets here are also intrinsically X-ray weak.

1. The soft stacked effective photon index $\left(1.8_{-0.4}^{+0.5}\right)$ for the sample (Section 4.1) argues against Compton-thick absorption in general. ${ }^{31}$ This is similar to the independent Chandra stacking results in L13 where we also found a relatively soft stacked signal for a subsample of high-redshift BAL quasars, which suggests that the stacked source is not Compton-thick but is intrinsically X-ray weak. A soft 3-24 keV effective photon index is also consistent with the lower energy Chandra or XMM-Newton spectral fitting results (based on $\approx 45-320$ X-ray photons) for IRAS 07598, PG 0946, PG 1001, and PG 1254 where no or only moderate $\left(\lesssim 10^{23} \mathrm{~cm}^{-2}\right)$ absorption was found (e.g., Sabra \& Hamann 2001; Imanishi \& Terashima 2004; Schartel et al. 2005; Saez et al. 2012). ${ }^{32}$

2. All six targets here and the two in the L13 pilot sample are significantly X-ray weak in the NuSTAR bands, which requires Compton-thick obscuration in the absorption scenario. If these BAL quasars with significant X-ray weakness represent an extension of the normal BAL-quasar population to higher column densities of $10^{23.5}-10^{25} \mathrm{~cm}^{-2}$, then a first order expectation would be that they might consist of both Compton-thin and Compton-thick objects, similar to

\footnotetext{
31 For the two targets in L13, PG 1004 has a soft effective photon index $(1.8 \pm 0.5)$ but it could be dominated by jet emission, and PG 1700 has a hard effective photon index but with a large uncertainty $(0.5 \pm 0.7)$. The nature of their hard X-ray weakness is not clear.

32 For the remaining two objects, PG 0043 is not detected by XMM-Newton and IRAS 14026 is weakly detected by Chandra and thus spectral analysis is not possible.
}

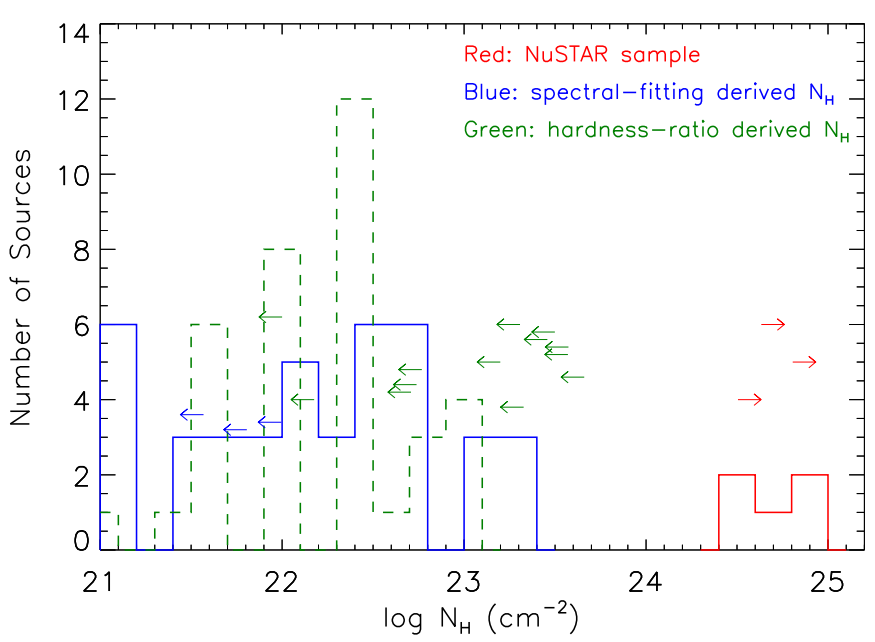

Figure 4. Distribution of the $N_{\mathrm{H}}$ constraints for the $N U S T A R$ BAL-quasar sample (red histogram and lower limits), assuming the observed hard X-ray weakness is caused by absorption. For comparison, $N_{\mathrm{H}}$ distributions for typical BAL quasars collected from the literature (Gallagher et al. 2002a; Giustini et al. 2008; Fan et al. 2009; Streblyanska et al. 2010; Morabito et al. 2014) are shown as the blue $\left(N_{\mathrm{H}}\right.$ derived from spectral fitting) and green $\left(N_{\mathrm{H}}\right.$ derived from hardnessratio analysis) histograms and upper limits. Sources with $N_{\mathrm{H}}<10^{21} \mathrm{~cm}^{-2}$ are included in the $N_{\mathrm{H}}=10^{21} \mathrm{~cm}^{-2}$ bin.

(A color version of this figure is available in the online journal.)

the overall AGN population. In this case, the chance of observing a sample of eight objects that are $100 \%$ Comptonthick is likely small. A natural explanation is that this is not a pure Compton-thick sample and at least some of the targets are intrinsically $\mathrm{X}$-ray weak.

In Figure 4, we show the $N_{\mathrm{H}}$ distributions for our $N u S T A R$ BAL-quasar sample (eight objects in total) ${ }^{33}$ and those BAL quasars collected from the literature (Gallagher et al. 2002a; Giustini et al. 2008; Fan et al. 2009; Streblyanska et al. 2010; Morabito et al. 2014). ${ }^{34}$ The combined distribution appears disjointed and perhaps bimodal, missing objects that are heavily obscured but Compton-thin, while the NUSTAR sample stands out in the Compton-thick regime forming an apparently distinct peak. The $N_{\mathrm{H}}$ distribution for typical low-redshift AGNs does not show such a bimodality (e.g., Figure 4 of Ueda et al. 2014). There is no obvious reason why BAL quasars would avoid the $N_{\mathrm{H}} \approx(5-20) \times 10^{23} \mathrm{~cm}^{-2}$ regime yet not higher $N_{\mathrm{H}}$ values, although we caution that our NuSTAR sample size is still relatively small and the apparent bimodality might be caused by small number statistics (moreover, we note that the shielding gas responsible for the X-ray absorption in BAL quasars is different from the dusty torus in typical obscured AGNs, and its nature is poorly understood). Thus, as a complement to the spectral-shape argument above, Figure 4 provides suggestive additional evidence that some of the NuSTAR objects are not absorbed by Compton-thick material but are intrinsically X-ray weak.

In obscured AGNs, there could be an additional soft $\mathrm{X}$-ray continuum component arising from electron scattering

\footnotetext{
33 The radio-loud nature of PG 1004 in L13 does not affect our analysis here. If its observed $\mathrm{X}$-ray emission has a significant jet-linked contribution, the estimated $N_{\mathrm{H}}$ value would be larger (see Section 4.1.1 of L13).

34 These are the X-ray studies of large samples of BAL quasars including $N_{\mathrm{H}}$ constraints in the literature. We caution that these data might not represent the real $N_{\mathrm{H}}$ distribution as the sample is not complete and the $N_{\mathrm{H}}$ constraints were obtained via different approaches.
} 
of the intrinsic continuum in an ionized medium surrounding the central engine on a larger scale than the X-ray absorber. The scattering zone has a very small column density so that the scattered continuum has approximately the same shape as the intrinsic continuum. ${ }^{35}$ Because of this, the scattered fraction is expected to be small, usually a few percent $(\approx 5 \%)$ or less (e.g., Turner et al. 1997; Ogle et al. 1999; Ueda et al. 2007; Young et al. 2007 and references therein). In principle, this ionized scattered component could perhaps dominate over the Compton-reflected component in the observed 3-24 keV emission of a Compton-thick AGN if the column density is sufficiently high $\left(N_{\mathrm{H}}>10^{25} \mathrm{~cm}^{-2}\right)$. In this case, the observed $\mathrm{X}$-ray spectrum would appear soft and also be hard X-ray weak by a factor of $\approx 20$ or more. This scenario could perhaps explain the soft stacked signal of our NUSTAR sample here without invoking intrinsic X-ray weakness. However, such a case would arguably be even more extraordinary than the discovery of intrinsically X-ray weak AGNs, as so far no compelling example of a clearly Compton-thick AGN with a soft $\approx 3-24 \mathrm{keV}$ continuum has been found (T. Yaqoob 2014, private communication). Furthermore, our X-ray variability detections for some objects (see Section 3) would constrain the size of any scattering medium. Therefore, we admit this possibility here but do not consider it likely.

Assuming that the observed hard X-ray weakness is entirely intrinsic with no absorption, these targets are intrinsically $\mathrm{X}$-ray weak from $3-24 \mathrm{keV}$ by the factors given in Table 2 $(\approx 4$ to $>25)$. If there is also absorption present that affects the observed 3-24 keV flux, similar to the case of Mrk 231, the factors of intrinsic X-ray weakness would be smaller. For comparison, Mrk 231 is intrinsically X-ray weak by a factor of $\approx 10$ after the absorption correction (Teng et al. 2014), and PG 1004 and PG 1700 in L13 are X-ray weak by about the same factor in the intrinsic X-ray weakness scenario. The underlying physics responsible for intrinsic X-ray weakness is still unclear; some sort of coronal-quenching mechanism might be relevant for BAL quasars (Section 4.2.1 of L13 and references therein).

There are two LoBAL and four HiBAL quasars in our sample (Section 5.1). We checked the stacked signals for these two groups of sources separately. For LoBAL quasars (IRAS 07598 and IRAS 14026), the stacked source is only weakly detected in the 3-8 keV band, and the lower limit on the effective photon index is 1.0 ( $>1.4$ at a $1 \sigma$ confidence level). For HiBAL quasars, the stacked effective photon index is $1.5_{-0.4}^{+0.4}$. Considering that the full sample has a soft effective photon index $\left(1.8_{-0.4}^{+0.5}\right)$, the LoBAL quasars likely have soft photon indices. It thus appears likely that both groups contain intrinsically X-ray weak quasars.

All three detected targets show significant flux variability in the 3-8 keV band when compared to earlier observations (Section 3). Usually we would not expect such significant variability in a Compton-thick AGN where the 3-8 keV spectrum is likely dominated by a Compton-reflected component, as variability would be washed out during reflection over an extended region. However, for our BAL-quasar targets here, the X-ray absorber (shielding gas) is located on a significantly smaller physical scale than the torus in typical obscured AGNs $\left(\approx 10^{16}\right.$ $10^{17} \mathrm{~cm}$ vs. parsec scale) and the variability observed is on multi-year timescales. It is possible that a reflection-dominated spectrum could show long-term variability (e.g., Matt et al.

\footnotetext{
35 The location and physical properties of the scattering medium in BAL quasars are uncertain. The shielding gas itself might produce a relatively soft scattered continuum if it is sufficiently highly ionized (e.g., Proga \& Kallman 2004; Ross \& Fabian 2005; García \& Kallman 2010).
}

2004), and the scenario of Compton-thick absorption cannot be excluded by such variability. Due to the limited photon statistics of the NUSTAR and/or previous Chandra and XMM-Newton data, we cannot constrain the variability of the spectral shape (e.g., $\left.\Gamma_{\text {eff }}\right)$ for these three targets.

The fraction of intrinsically X-ray weak quasars among our sample objects is likely high. The soft stacked signal is not dominated by one single object, and a lower limit on the fraction is $33 \%$ (at least two out of six being intrinsically X-ray weak) with an upper limit of $100 \%$ (all being intrinsically X-ray weak). Our targets were selected to be significantly X-ray weak in the $<10 \mathrm{keV}$ band, and thus the intrinsic X-ray weakness fraction among the general BAL-quasar population will be lower. The fraction is estimated to be $\approx 17-40 \%$ in the LBQS BAL-quasar sample (L13), much larger than the $\lesssim 2 \%$ fraction among nonBAL quasars (Gibson et al. 2008). L13 suggested that the disk wind in an intrinsically X-ray weak quasar might have a large covering factor as it is likely easier to launch the wind when the nuclear X-ray emission is weak. ${ }^{36}$ Thus intrinsically X-ray weak quasars would be preferentially observed as BAL quasars.

\section{SUMMARY AND FUTURE WORK}

We have presented NUSTAR observations of an extended sample of six BAL quasars with significant X-ray weakness in the $<10 \mathrm{keV}$ band. All targets are either marginally or not detected by $N U S T A R$, indicating significant hard X-ray (8-24 keV) weakness as well, similar to the pilot sample in L13. The derived column-density constraints in an absorption scenario are all in the Compton-thick regime. However, stacking and joint spectral analyses of the data indicate a soft effective photon index, generally disfavoring Compton-thick absorption. Moreover, the uniform hard X-ray weakness observed in this sample and also the pilot sample suggests that the X-ray weakness is intrinsic in at least some of the targets. We conclude that NuSTAR observations of BAL-quasar samples have likely discovered a significant population $(\gtrsim 33 \%)$ of intrinsically $\mathrm{X}$-ray weak sources among the BAL quasars with significantly weak $<10 \mathrm{keV}$ emission. We emphasize that the disk wind in an intrinsically X-ray weak quasar might have a large covering factor, and thus the source would be preferentially observed as an BAL quasar.

It would be worthwhile to obtain additional $N U S T A R$ observations of our targets, so that better constraints can be derived on the spectral shapes of individual and stacked sources. For example, by tripling the exposure times, the three undetected sources should in general be detected in the $3-8 \mathrm{keV}$ band and the stacked source of these three will probably be detected in the 8-24 keV band, as suggested by the current stacking results. Meanwhile, PG 1001 and PG 1254 should likely be individually detected in the 8-24 keV band. The improved spectral-shape constraints would provide a tighter constraint on the fraction of intrinsically X-ray weak quasars among this sample. Moreover, by increasing the exposure times by factors of $\approx 5-10$, sufficient photon statistics could probably be obtained for some individual targets to allow identification of intrinsically X-ray weak quasars in the sample via basic spectral analysis and accurate measurements of a soft $\Gamma_{\text {eff }}$. Such longer observations could be divided into a few segments to probe any short-term variability of the targets; ideally these segments should be separated

\footnotetext{
36 Intrinsic X-ray weakness might also be associated with powerful large-scale outflows as suggested by Teng et al. (2014).
} 
by somewhat less than the expected light-crossing time of the shielding gas $(\approx 4-40$ days in the rest frame).

It would also be valuable to select additional intrinsically $\mathrm{X}$ ray weak candidates for $N u S T A R$ observations, although sources satisfying the selection criteria in Section 2 are rare largely due to the lack of systematic BAL-quasar selection at low redshifts $(z \lesssim 1.4)$, where the key C IV transition is generally not accessible via ground-based spectroscopy. Such studies could probably also be extended to include "mini-BAL" quasars, which have narrower absorption troughs (500-2000 $\mathrm{km} \mathrm{s}^{-1}$ wide) than BAL quasars yet may share their other properties (e.g., Trump et al. 2006).

Presently, the fraction of BAL quasars that are intrinsically X-ray weak is poorly constrained in the LBQS sample $(\approx 17 \%-40 \%$; L13), which was derived via stacking analysis of a sample of Chandra 2-8 keV undetected objects. We have scheduled additional 9-12 ks Chandra observations of the six undetected HiBAL quasars in the sample. Based on our current Chandra stacking results, we suspect that these observations will convert most of these non-detections into detections. This will set a much tighter and more robust upper limit upon the fraction of HiBAL quasars that are intrinsically X-ray weak. Further X-ray observations of the undetected LoBAL quasars in the sample may be pursued in future work. Such studies will benefit our assessment of the different fractions of intrinsically $\mathrm{X}$-ray weak objects among BAL and non-BAL quasars.

We acknowledge support from the California Institute of Technology (Caltech) NuSTAR subcontract 44A-1092750 (B.L., W.N.B.), NASA ADP grant NNX10AC99G (B.L., W.N.B.), NASA Postdoctoral Program (S.H.T.), CONICYTChile FONDECYT 1140304 (P.A.) and 1141218 (F.E.B.), "EMBIGGEN" Anillo ACT1101 (P.A., F.E.B.), Basal-CATA PFB-06/2007 (F.E.B.), Project IC120009 "Millennium Institute of Astrophysics (MAS)" of Iniciativa Científica Milenio del Ministerio de Economía, Fomento y Turismo (F.E.B.), ASI/INAF grant I/037/12/0-011/13 (A.C.), STFC grant ST/J003697/1 (P.G.), and the Swiss National Science Foundation (NSF) grant PP00P2 138979/1 (M.K.). We thank K. Forster for help with the observation planning, and we thank T. Yaqoob for helpful discussions. We thank the referee for carefully reviewing the manuscript and providing helpful comments.

This work was supported under NASA Contract No. NNG08FD60C, and made use of data from the NuSTAR mission, a project led by Caltech, managed by the Jet Propulsion Laboratory, and funded by the National Aeronautics and Space Administration. We thank the NUSTAR Operations, Software and Calibration teams for support with the execution and analysis of these observations. This research has made use of NuSTARDAS jointly developed by the ASI Science Data Center (ASDC, Italy) and Caltech (USA).

\section{REFERENCES}

Alexander, D. M., Stern, D., Del Moro, A., et al. 2013, ApJ, 773, 125 Allen, J. T., Hewett, P. C., Maddox, N., Richards, G. T., \& Belokurov, V. 2011, MNRAS, 410, 860

Arav, N., de Kool, M., Korista, K. T., et al. 2001, ApJ, 561, 118

Arav, N., Korista, K. T., de Kool, M., et al. 1999, ApJ, 516, 27

Arnaud, K. A. 1996, in ASP Conf. Ser., Vol. 101, Astronomical Data Analysis Software and Systems V, ed. G. H. Jacoby \& J. Barnes (San Francisco, CA: ASP), 17

Ballo, L., Piconcelli, E., Schartel, N., \& Vignali, C. 2008, arXiv:0807.2225

Baumgartner, W. H., Tueller, J., Markwardt, C. B., et al. 2013, ApJS, 207, 19

Becker, R. H., White, R. L., \& Helfand, D. J. 1995, ApJ, 450, 559
Brandt, W. N., Laor, A., \& Wills, B. J. 2000, ApJ, 528, 637

Cardelli, J. A., Clayton, G. C., \& Mathis, J. S. 1989, ApJ, 345, 245

Comastri, A., Ranalli, P., Iwasawa, K., et al. 2011, A\&A, 526, L9

Condon, J. J., Cotton, W. D., Greisen, E. W., et al. 1998, AJ, 115, 1693

Dickey, J. M., \& Lockman, F. J. 1990, ARA\&A, 28, 215

Fan, L. L., Wang, H. Y., Wang, T., et al. 2009, ApJ, 690, 1006

Freeman, P. E., Kashyap, V., Rosner, R., \& Lamb, D. Q. 2002, ApJS, 138, 185

Gallagher, S. C., Brandt, W. N., Chartas, G., \& Garmire, G. P. 2002a, ApJ, 567,37

Gallagher, S. C., Brandt, W. N., Chartas, G., Garmire, G. P., \& Sambruna, R. M. 2002b, ApJ, 569, 655

Gallagher, S. C., Brandt, W. N., Chartas, G., et al. 2006, ApJ, 644, 709

Gallagher, S. C., Brandt, W. N., Laor, A., et al. 2001, ApJ, 546, 795

Gallagher, S. C., Brandt, W. N., Sambruna, R. M., Mathur, S., \& Yamasaki, N. 1999, ApJ, 519, 549

Gallagher, S. C., Brandt, W. N., Wills, B. J., et al. 2004, ApJ, 603, 425

Gandhi, P., Lansbury, G. B., Alexander, D. M., et al. 2014, ApJ, 792, 117

García, J., \& Kallman, T. R. 2010, ApJ, 718, 695

Gehrels, N. 1986, ApJ, 303, 336

George, I. M., \& Fabian, A. C. 1991, MNRAS, 249, 352

Ghisellini, G., Haardt, F., \& Matt, G. 1994, MNRAS, 267, 743

Gibson, R. R., \& Brandt, W. N. 2012, ApJ, 746, 54

Gibson, R. R., Brandt, W. N., \& Schneider, D. P. 2008, ApJ, 685, 773

Gibson, R. R., Jiang, L., Brandt, W. N., et al. 2009, ApJ, 692, 758

Giustini, M., Cappi, M., \& Vignali, C. 2008, A\&A, 491, 425

Green, P. J., Aldcroft, T. L., Mathur, S., Wilkes, B. J., \& Elvis, M. 2001, ApJ, 558,109

Haardt, F., \& Maraschi, L. 1991, ApJL, 380, L51

Hamann, F. 1998, ApJ, 500, 798

Hao, C. N., Xia, X. Y., Mao, S., Wu, H., \& Deng, Z. G. 2005, ApJ, 625, 78

Harrison, F. A., Craig, W. W., Christensen, F. E., et al. 2013, ApJ, 770, 103

Hewett, P. C., \& Foltz, C. B. 2003, AJ, 125, 1784

Hines, D. C., Schmidt, G. D., Gordon, K. D., et al. 2001, ApJ, 563, 512

Hines, D. C., \& Wills, B. J. 1995, ApJL, 448, L69

Imanishi, M., \& Terashima, Y. 2004, AJ, 127, 758

Jiang, P., Zhou, H., Ji, T., et al. 2013, AJ, 145, 157

Just, D. W., Brandt, W. N., Shemmer, O., et al. 2007, ApJ, 665, 1004

Kellermann, K. I., Sramek, R., Schmidt, M., Shaffer, D. B., \& Green, R. 1989, AJ, 98, 1195

Komatsu, E., Smith, K. M., Dunkley, J., et al. 2011, ApJS, 192, 18

Kraft, R. P., Burrows, D. N., \& Nousek, J. A. 1991, ApJ, 374, 344

Lansbury, G. B., Alexander, D. M., Del Moro, A., et al. 2014, ApJ, 785, 17

Lehmer, B. D., Alexander, D. M., Bauer, F. E., et al. 2010, ApJ, 724, 559

Leighly, K. M., Halpern, J. P., Jenkins, E. B., \& Casebeer, D. 2007a, ApJS, 173,1

Leighly, K. M., Halpern, J. P., Jenkins, E. B., et al. 2007b, ApJ, 663, 103

Luo, B., Brandt, W. N., Alexander, D. M., et al. 2013, ApJ, 772, 153 (L13)

Lusso, E., Comastri, A., Vignali, C., et al. 2010, A\&A, 512, A34

Martin, D. C., Fanson, J., Schiminovich, D., et al. 2005, ApJL, 619, L1

Mathur, S., Green, P. J., Arav, N., et al. 2000, ApJL, 533, L79

Matt, G., Bianchi, S., Guainazzi, M., \& Molendi, S. 2004, A\&A, 414, 155

Matt, G., Brandt, W. N., \& Fabian, A. C. 1996, MNRAS, 280, 823

Miller, B. P., Brandt, W. N., Gallagher, S. C., et al. 2006, ApJ, 652, 163

Miller, B. P., Brandt, W. N., Schneider, D. P., et al. 2011, ApJ, 726, 20

Morabito, L. K., Dai, X., Leighly, K. M., Sivakoff, G. R., \& Shankar, F. 2014, ApJ, 786, 58

Murphy, K. D., \& Yaqoob, T. 2009, MNRAS, 397, 1549

Murray, N., Chiang, J., Grossman, S. A., \& Voit, G. M. 1995, ApJ, 451, 498

O’Donnell, J. E. 1994, ApJ, 422, 158

Ogle, P. M., Cohen, M. H., Miller, J. S., et al. 1999, ApJS, 125, 1

Park, T., Kashyap, V. L., Siemiginowska, A., et al. 2006, ApJ, 652, 610

Proga, D., \& Kallman, T. R. 2004, ApJ, 616, 688

Proga, D., Stone, J. M., \& Kallman, T. R. 2000, ApJ, 543, 686

Richards, G. T., Lacy, M., Storrie-Lombardi, L. J., et al. 2006, ApJS, 166, 470

Ross, R. R., \& Fabian, A. C. 2005, MNRAS, 358, 211

Rovilos, E., Georgantopoulos, I., Akylas, A., et al. 2014, MNRAS, 438, 494

Sabra, B. M., \& Hamann, F. 2001, ApJ, 563, 555

Saez, C., Brandt, W. N., Gallagher, S. C., Bauer, F. E., \& Garmire, G. P. 2012, ApJ, 759, 42

Schartel, N., Rodríguez-Pascual, P. M., Santos-Lleó, M., et al. 2005, A\&A, 433, 455

Schmidt, M., \& Green, R. F. 1983, ApJ, 269, 352

Schneider, D. P., Richards, G. T., Hall, P. B., et al. 2010, AJ, 139, 2360

Shen, Y., \& Liu, X. 2012, ApJ, 753, 125

Shen, Y., Richards, G. T., Strauss, M. A., et al. 2011, ApJS, 194, 45

Skrutskie, M. F., Cutri, R. M., Stiening, R., et al. 2006, AJ, 131, 1163

Sprayberry, D., \& Foltz, C. B. 1992, ApJ, 390, 39 
Steffen, A. T., Strateva, I., Brandt, W. N., et al. 2006, AJ, 131, 2826

Streblyanska, A., Barcons, X., Carrera, F. J., \& Gil-Merino, R. 2010, A\&A, 515, A2

Teng, S. H., Brandt, W. N., Harrison, F. A., et al. 2014, ApJ, 785, 19

Trump, J. R., et al. 2006, ApJS, 165, 1

Turner, T. J., George, I. M., Nandra, K., \& Mushotzky, R. F. 1997, ApJ, 488,164

Turnshek, D. A., Espey, B. R., Kopko, M., Jr., et al. 1994, ApJ, 428, 93

Ueda, Y., Akiyama, M., Hasinger, G., Miyaji, T., \& Watson, M. G. 2014, ApJ, 786,104
Ueda, Y., Eguchi, S., Terashima, Y., et al. 2007, ApJL, 664, L79

Vanden Berk, D. E., Richards, G. T., Bauer, A., et al. 2001, AJ, 122, 549

Weymann, R. J., Morris, S. L., Foltz, C. B., \& Hewett, P. C. 1991, ApJ, 373, 23

Wik, D. R., Hornstrup, A., Molendi, S., et al. 2014, ApJ, 792, 48

Wright, E. L., Eisenhardt, P. R. M., Mainzer, A. K., et al. 2010, AJ, 140,1868

Wu, J., Brandt, W. N., Hall, P. B., et al. 2011, ApJ, 736, 28

York, D. G., Adelman, J., Anderson, J. E., Jr., et al. 2000, AJ, 120, 1579

Young, S., Axon, D. J., Robinson, A., Hough, J. H., \& Smith, J. E. 2007, Natur, 450,74 